\title{
Application of GPS in geomagnetic survey
}

\author{
Zuowen Gu, Zhijia Zhan, Jintian Gao, Tongqi Yao, and Wei Han \\ Institute of Geophysics, China Earthquake Administration, Beijing 100081, China
}

(Received April 22, 2005; Revised August 26, 2005; Accepted August 26, 2005; Online published June 2, 2006)

\begin{abstract}
When measuring the geomagnetic declination, the azimuth of the reference mark must be measured. This paper briefly describes the principle of measuring the azimuth by the differential GPS (Global Positioning System), several test observations of GPS and the results. In geomagnetic survey in China during 2002 2003, GPS was used in measuring the azimuth. Field observations by GPS were carried out during the period that satellite signals were better received. The statistical results of the GPS data at 135 stations in China show that the difference of the azimuths between 2 GPS measurements at various stations is $0.0^{\prime \prime} \sim 5.9^{\prime \prime}$ and the average is $1.6^{\prime \prime}$. The azimuthal accuracy measured by GPS in this paper is $0.1^{\prime}$, and it is much better than the azimuthal accuracy $1.0^{\prime}$ given by Newitt et al. (1996). The observation results show that the azimuths measured by GPS are accurate and reliable; they are not related to the time-space changes. The geomagnetic declinations $D$ at various stations were obtained based on the azimuths measured by GPS, and the standard deviation of the reduced value of $D$ was $<0.4^{\prime}$. Using the reduced values, the 8-order spherical cap harmonic model and the 5-order Taylor polynomial model in China for $D$ were obtained. In geomagnetic survey, GPS is also used in measuring the longitude, the latitude and the elevation at various stations.
\end{abstract}

Key words: Geomagnetic survey, GPS, azimuth, geomagnetic declination, geomagnetic field model, China.

\section{Introduction}

Geomagnetic field is one of the important geophysical fields. Geomagnetic survey can monitor the electromagnetic environment of the earth on which people depend for existence. Geomagnetic data are widely applied in many fields, such as environmental protection, resource exploration, scientific research, geological structure, earthquake and volcanic eruption prediction, and space weather etc.

Geomagnetic field is a vector field that changes with time and space. To describe geomagnetic field needs to observe three components of geomagnetic field, which are mutually independent. Usually, there are 7 elements to express the geomagnetic field: the geomagnetic total intensity $F$, the declination $D$, the inclination $I$, the north component $X$, the east component $Y$, the horizontal component $H$ and the vertical component $Z$. In geomagnetic observations with 3 mutually independent components, $D$ must be measured, because no method exists for measuring the east component $Y$ that also contains the azimuthal information. In determining $D$ with the aid of the reference mark, the DI magnetometer is used to measure $D_{1}$, which is an angle between the geomagnetic north direction and the reference mark. The traditional astronomical observation is used to measure the azimuth $D_{2}$, which is an angle between the geographical north direction and the reference mark; then geomagnetic declination $D=D_{1}-D_{2}$.

The traditional method for measuring the azimuth is the astronomical observation, which uses the theodolite to ob-

Copyright (c) The Society of Geomagnetism and Earth, Planetary and Space Sciences (SGEPSS); The Seismological Society of Japan; The Volcanological Society of Japan; The Geodetic Society of Japan; The Japanese Society for Planetary Sciences; TERRAPUB. serve the Sun or observe the Pole Star. The astronomical method is accurate and involves less hardware and software. However, the astronomical observation requires large work load and is much influenced by the weather (Jankowski and Sucksdoff, 1996). The International Association of Geomagnetism and Aeronomy (IAGA) suggested and recommended to use the differential GPS to measure the azimuth (Newitt et al., 1996).

GPS has the advantages of small equipment volume, high observation accuracy, fast observation speed, all-weather operation, intelligent record, and software data processing etc. Therefore, GPS is widely used in many fields, such as geodesy, geophysics, geodynamics, geology, geomagnetism and seismology etc. GPS has become an important tool in monitoring and studying the global plate movement, the tectonic activity, the crust deformation and the earthquake precursor (Ma et al., 2001; Yu et al., 2003; Zhu et al., 2003; Wang et al., 2004; Koketsu et al., 1998; Nishimura et al., 2004; Hu et al., 2001; Miura et al., 2000; Hori et al., 2000; McClusky et al., 2003; Caporali et al., 2003; Nocquel and Calais, 2004; Fernandez et al., 2004).

GPS is a new technology in geomagnetic survey. What is the principle of measuring the azimuth by GPS? What is the better condition of measuring the azimuth by GPS? How is the azimuthal accuracy measured by GPS? These problems are very important and should be studied.

This paper describes the principle of measuring the azimuth by GPS. In order to examine the applicability of the GPS method to absolute geomagnetic measurement, we have carried out many GPS test observations in a deep investigation and research. In geomagnetic survey, GPS is used in measuring the azimuth, as well as the longitude, the 


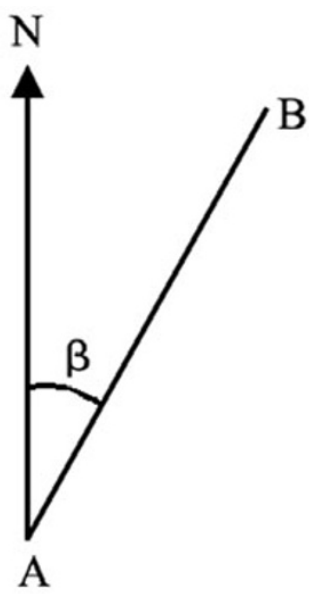

(a)

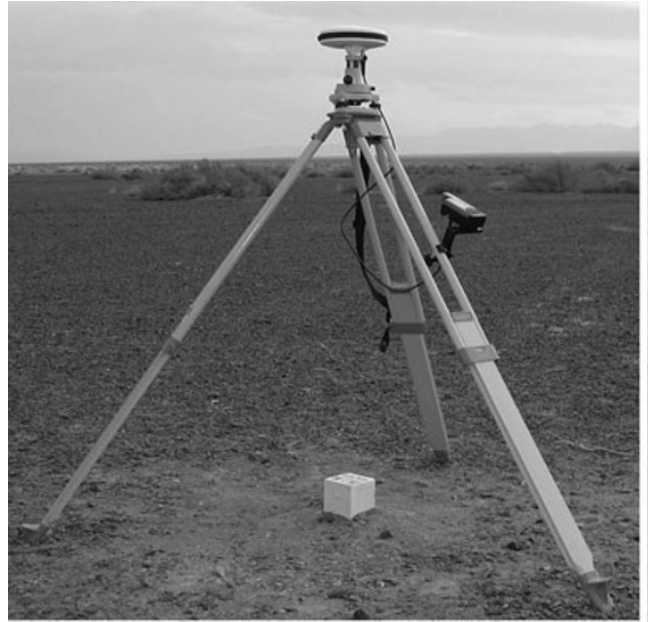

A

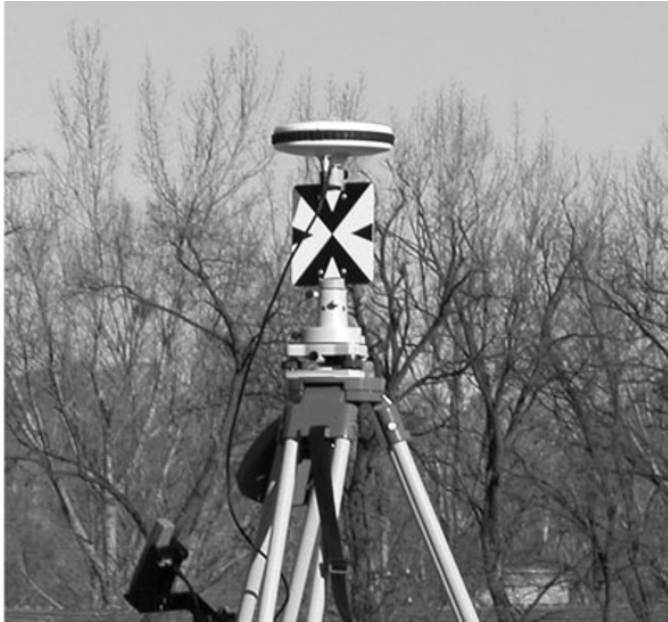

B

(b)

Fig. 1. (a) Configuration of measuring the azimuth by GPS. The point A represents geomagnetic station, the point B represents reference mark, N represents the north direction, $\beta$ represents the angle between the base-line AB and the north direction N. A typical distance between $\mathrm{A}$ and $\mathrm{B}$ is $250 \mathrm{~m}$. (b) Photos of GPS at the point A (geomagnetic station) and GPS with a markerat the point B (reference mark).

latitude and the elevation at various stations.

\section{Principle of Measuring the Azimuth by GPS}

The principle of measuring the azimuth by GPS is the same as that of the relative location. In Fig. 1, we set up two GPS receivers at point A (the geomagnetic station or site) and point B (the reference mark) respectively for synchronous measurements, which is called the differential GPS. The base-line vector $\mathrm{AB}$, the longitude, the latitude and the elevation at points $\mathrm{A}$ and $\mathrm{B}$ are obtained from the GPS observational data. Therefore, the angle $\beta$ between the base-line $\mathrm{AB}$ and the north direction $\mathrm{N}$ can be calculated (Fig. 1).

The geodetical azimuth $\beta$ on the elliptical surface can be directly derived from point A coordinate $\left(x_{1}, y_{1}\right)$ and point B coordinate $\left(x_{2}, y_{2}\right)$ :

$$
\beta=\arctan \left(x_{2}-x_{1}\right) /\left(y_{2}-y_{1}\right)
$$

where $x_{1}, y_{1}$ and $x_{2}, y_{2}$ are in the geodetical coordinate system (Kong and Mei, 1996).

In order to change the geodetical azimuth $\beta$ on the elliptical surface into the azimuth $\alpha$ on the local Cartesian coordinate system, the convergence angle $\gamma$ on the plane meridian and the direction correction $\delta$ must be taken into account (Kong and Mei, 1996):

$$
\alpha=\beta-\gamma+\delta
$$

The convergence angle $\gamma$ on the plane meridian can be expressed approximately as (Kong and Mei, 1996):

$$
\gamma=\lambda \sin \varphi+\lambda^{3} \sin \varphi \cos ^{2} \varphi\left(1+3 \eta^{2}+2 \eta^{4}\right) / 3
$$

where $\eta^{2}=e \cos ^{2} \varphi, \lambda$ is the difference of longitude and $\varphi$ is the latitude, $e$ is the second elliptical eccentricity $e=$ $\left(a^{2}-b^{2}\right)^{1 / 2} / b, a$ is the semi-major axis of the ellipsoid and $b$ is the semi-minor axis.
The direction correction $\delta$ can be approximately expressed as (Kong and Mei, 1996):

$$
\delta=P /\left(2 R^{2}\right)
$$

where $P$ is the pattern area on the ellipsoid surface, $R$ is the earth's radius, the unit of the direction correction $\delta$ in formula (4) is one second of arc.

Based on the principle of measuring the azimuth by GPS, the related software was compiled and provided by the Ashtech (Ashtech Solutions Software). This software can accurately process the data of GPS observation and calculate the azimuth.

\section{Test Results of Measuring the Azimuth by GPS 3.1 Ashtech ProMark 2 GPS system}

In geomagnetic survey, the GPS equipment used is the Ashtech ProMark 2 GPS system made in the USA. The main functions of the GPS are: GPS satellite tracking, 10 independent parallel channels, L1C/A all-carrier phase, WAAS/EGNOS satellite tracking, 2 independent parallel channels; the accuracy indexes are: static measurement parameters (RMS) are $5 \mathrm{~mm}+1 \mathrm{ppm}$ for horizontal, 10 $\mathrm{mm}+2 \mathrm{ppm}$ for vertical and $<0.1^{\prime}$ for the azimuth. The observation time is $20 \sim 60$ min depending on the length of the baseline and the condition of the satellite. The main functions of GPS software are: the software interface is good after solutions, the operation is easy and can accurately process the observational data of GPS, it has friendly users' interface for Windows.

GPS is used in measuring the azimuth so as to determine the geomagnetic declination. Moreover, GPS is also used in measuring the longitude, the latitude and the elevation at stations and sites, as well as locating the work points and searching the stations and sites.

\subsection{Test results of measuring the azimuth by GPS}

In order to examine the applicability of the GPS method to absolute geomagnetic measurement, several test observations of measuring the azimuth by GPS were carried out in 
Table 1. Comparison between the azimuths $\alpha$ measured by GPS and $\beta$ measured by the astronomical method in 2002 .

\begin{tabular}{ccccc}
\hline Date & \multicolumn{2}{c}{ Jan. $31 \sim$ Feb. 1} & Feb. $28 \sim$ March 1 & March 12 \\
\hline Place & \multicolumn{2}{c}{ Jinghai observatory } & Shisanling observatory & Shisanling observatory \\
L $(\mathrm{m})$ & 165.896 & 150.294 & 223.920 & 223.920 \\
$\alpha$ & $89^{\circ} 39^{\prime} 20.2^{\prime \prime}$ & $15^{\circ} 16^{\prime} 20.2^{\prime \prime}$ & $115^{\circ} 43^{\prime} 56.5^{\prime \prime}$ & $115^{\circ} 43^{\prime} 56.6^{\prime \prime}$ \\
$\beta$ & $89^{\circ} 39^{\prime} 19.5^{\prime \prime}$ & $15^{\circ} 16^{\prime} 18.3^{\prime \prime}$ & $115^{\circ} 43^{\prime} 52.8^{\prime \prime}$ & $115^{\circ} 43^{\prime} 52.8^{\prime \prime}$ \\
$\Delta \alpha$ & $0.7^{\prime \prime}$ & $1.9^{\prime \prime}$ & $3.7^{\prime \prime}$ & $3.8^{\prime \prime}$ \\
\hline
\end{tabular}

Table 2. Comparison between the GPS method and the astronomical method.

\begin{tabular}{lll}
\hline Method & Major advantages & Major disadvantages \\
\hline GPS & $\begin{array}{l}\text { 1. Automatic observation; } \\
\text { 2. Few influence of weather. }\end{array}$ & $\begin{array}{l}\text { 1. Price of the equipment is higher; } \\
\text { 2. Must set up GPS at the reference mark. }\end{array}$ \\
Astronomical & $\begin{array}{l}\text { 1. Price of the equipment is lower; } \\
\text { 2. Can select a far object }\end{array}$ & 1. Artificial observation only; \\
& as the reference mark. Much influence of weather.
\end{tabular}

Table 3. Difference $\Delta \alpha_{1}$ between the azimuths $\alpha\left(207^{\circ} 35^{\prime}+\Delta \alpha_{1}\right)$ and the average measured by 10 sets of GPS and the difference $\Delta \alpha_{2}$ in two measurements by each set of GPS.

\begin{tabular}{cccccc}
\hline GPS (set) & 1 & 2 & 3 & 4 & 5 \\
\hline$\alpha_{1}$ & $52.4^{\prime \prime}$ & $55.1^{\prime \prime}$ & $50.5^{\prime \prime}$ & $54.7^{\prime \prime}$ & $54.0^{\prime \prime}$ \\
$\Delta \alpha_{1}$ & $-0.3^{\prime \prime}$ & $2.4^{\prime \prime}$ & $-2.2^{\prime \prime}$ & $2.0^{\prime \prime}$ & $1.3^{\prime \prime}$ \\
$\Delta \alpha_{2}$ & $0.1^{\prime \prime}$ & $3.4^{\prime \prime}$ & $3.0^{\prime \prime}$ & $5.1^{\prime \prime}$ & $1.6^{\prime \prime}$ \\
\hline GPS (set) & 6 & 7 & 8 & 9 & 10 \\
\hline$\alpha_{1}$ & $51.3^{\prime \prime}$ & $49.5^{\prime \prime}$ & $52.6^{\prime \prime}$ & $52.6^{\prime \prime}$ & $54.8^{\prime \prime}$ \\
$\Delta \alpha_{1}$ & $-1.4^{\prime \prime}$ & $-3.2^{\prime \prime}$ & $-0.1^{\prime \prime}$ & $-0.1^{\prime \prime}$ & $2.1^{\prime \prime}$ \\
$\Delta \alpha_{2}$ & $4.2^{\prime \prime}$ & $3.4^{\prime \prime}$ & $5.1^{\prime \prime}$ & $0.3^{\prime \prime}$ & $0.9^{\prime \prime}$ \\
\hline
\end{tabular}

a deep investigation and research. Table 1 shows a comparison between the test results in measuring the azimuths by GPS and those by astronomical method in 2002. In Table 1, the baseline length $L$ represents the distance between the site and the reference mark; $\alpha$ represents the azimuth measured by GPS; $\beta$ represents the azimuth measured by the astronomical observation, this azimuth is determined from the pole star observation; $\Delta \alpha$ expresses the difference $(\alpha-$ $\beta$ ) between the azimuths obtained by the two methods. It can be seen in Table 1 that $\Delta \alpha$ is $0.7^{\prime \prime} \sim 3.8^{\prime \prime}$, the mean is $2.5^{\prime \prime}$ and the standard deviation is $1.5^{\prime \prime}$; the differences are very small. The results of astronomical observations show that an estimation of accuracy for the azimuth obtained by astronomical method is $2^{\prime \prime} \sim 3^{\prime \prime}$ (Zhou et al., 1997). It is seen that the GPS method has the similar accuracy as the astronomical method for measuring the azimuth. Therefore, the GPS method of measuring the azimuth can replace the traditional astronomical method.

Table 2 lists major advantages and disadvantages of measuring the azimuth by the GPS method and the astronomical method. We would suggest to use the GPS method if possible, because GPS method is automatic observation and can greatly decrease the influence of weather on the geomag- netic survey.

Other tests were done for 10 sets of GPS at Shisanling Observatory in Beijing during December 4 6, 2003. The test operations were carried out at the site mark $\mathrm{A}$ and the reference mark B. Both were $20 \times 20 \times 80 \mathrm{~cm}^{3}$ marble marks, and the buried depth was $75 \mathrm{~cm}$. The separation between the two marks was $236 \mathrm{~m}$. During the test period, GPS was at good working condition, the number of the received satellite was $5 \sim 10$, and most of them were $6 \sim 8$. The PDOP (Positioning Dilution of Precision) value was 1.9 3.0. The observation time of GPS was $20 \sim 60$ min, and most of them were $30 \sim 40 \mathrm{~min}$. The test results show the average separation between mark A and mark B is $236.1147 \mathrm{~m}$, and the average azimuth is $207^{\circ} 35^{\prime} 52.7^{\prime \prime}$. In Table 3, $\Delta \alpha_{1}$ represents the difference between the azimuths and the average, and $\Delta \alpha_{2}$ represents the difference between the azimuths in two measurements by GPS. It can be seen in Table 3 that the difference $\Delta \alpha_{2}$ of the azimuths measured in two measurements by each set of GPS is $0.1^{\prime \prime} \sim 5.1^{\prime \prime}$, the mean is $2.7^{\prime \prime}$ and the standard deviation is $1.9^{\prime \prime}$. The difference $\Delta \alpha_{1}$ between the azimuths and the average for each set of GPS is $-3.2^{\prime \prime} \sim 2.4^{\prime \prime}$, the mean of absolute difference is $1.5^{\prime \prime}$ and the standard deviation is 
Table 4. The azimuths $\alpha$ measured by GPS at Shisanling Observatory and the difference $\Delta \alpha$ between the azimuths and the average $\left(115^{\circ} 44^{\prime} 0.2^{\prime \prime}\right)$ in different time during 2002 2003.

\begin{tabular}{|c|c|c|c|c|c|}
\hline \multirow[t]{2}{*}{ Time } & \multicolumn{2}{|l|}{2002} & \multicolumn{3}{|c|}{2003} \\
\hline & Feb. 28 Mar. 1 & March 12 & April 15 & Feb. 26 & Sept. 26 \\
\hline $\mathrm{M}$ & 6 & 5 & 8 & 8 & 5 \\
\hline$\alpha$ & $115^{\circ} 43^{\prime} 56.5^{\prime \prime}$ & $115^{\circ} 43^{\prime} 56.6^{\prime \prime}$ & $115^{\circ} 44^{\prime} 0.6^{\prime \prime}$ & $115^{\circ} 44^{\prime} 3.7^{\prime \prime}$ & $115^{\circ} 44^{\prime} 3.7^{\prime \prime}$ \\
\hline$\Delta \alpha$ & $-3.7^{\prime \prime}$ & $-3.6^{\prime \prime}$ & $0.4^{\prime \prime}$ & $3.5^{\prime \prime}$ & $3.5^{\prime \prime}$ \\
\hline
\end{tabular}

Table 5. Statistical results of the observation time $t(\mathrm{~min})$ by GPS, the number Ns of the received satellite and the PDOP value in different regions of China.

\begin{tabular}{|c|c|c|c|c|}
\hline Region & M & $\mathrm{t}(\min )$ & Ns & PDOP \\
\hline \multirow[t]{2}{*}{ Northeast region } & 18 & $35 \sim 103$ & $5 \sim 9$ & $1.8 \sim 4.5$ \\
\hline & & $52.9 \pm 21.8$ & $7.1 \pm 0.8$ & $2.6 \pm 0.6$ \\
\hline \multirow[t]{2}{*}{ North-China region } & 18 & $22 \sim 77$ & $5 \sim 10$ & $1.9 \sim 5.4$ \\
\hline & & $39.9 \pm 14.4$ & $7.4 \pm 0.9$ & $2.6 \pm 0.7$ \\
\hline \multirow[t]{2}{*}{ East-China region } & 20 & $21 \sim 82$ & $5 \sim 10$ & $1.9 \sim 4.8$ \\
\hline & & $42.1 \pm 15.5$ & $7.4 \pm 0.9$ & $2.8 \pm 0.7$ \\
\hline \multirow[t]{2}{*}{ Southwest region } & 38 & $35 \sim 95$ & $5 \sim 10$ & $1.8 \sim 4.5$ \\
\hline & & $55.9 \pm 19.0$ & $7.1 \pm 1.0$ & $2.9 \pm 0.7$ \\
\hline \multirow[t]{2}{*}{ Northwest region } & 41 & $18 \sim 70$ & $6 \sim 10$ & $1.7 \sim 4.4$ \\
\hline & & $36.2 \pm 13.4$ & $7.7 \pm 1.6$ & $2.6 \pm 0.7$ \\
\hline
\end{tabular}

$1.1^{\prime \prime}$. The results show that the functions of 10 sets of GPS are good.

Table 4 shows the difference $\Delta \alpha$ between the azimuths $\alpha$ measured by GPS and the average in different time at Shisanling Observatory in Beijing during 2002 2003. In Table 4, M is the number of GPS observations. All of the test work were carried out at the fixed marks A and B. The separation between these two marks was $236 \mathrm{~m}$. GPS was in good working condition. It can be seen from Table 4 that the azimuths $\alpha$ measured by GPS were quite stable, and the difference $\Delta \alpha$ between the azimuths and the average value was $-3.7^{\prime \prime} \sim 3.5^{\prime \prime}$, the mean of the absolute differences was $2.9^{\prime \prime}$ and the standard deviation was $1.4^{\prime \prime}$. The results show that the azimuths measured by GPS are stable and accurate; they are not related to the time change.

\section{GPS Field Measurement}

\subsection{Location of geomagnetic station}

Using GPS, the longitude, the latitude and the elevation at 135 stations in China were measured very well. The results show that the mean standard deviations of the longitude and the latitude are $0.053^{\prime \prime}$ and $0.078^{\prime \prime}$ respectively; the mean standard deviation of the elevation at the stations is $3.79 \mathrm{~m}$.

\subsection{Measuring the azimuth by GPS}

The measurements at various stations include the geomagnetic three-component and the GPS observations. In the geomagnetic survey, we ensured the position of G-856 magnetometer's sensor at the same place with that of the DI magnetometer's coil, and we ensured the centralization position of DI magnetometer at the same place with that of the GPS.

The azimuth of the reference mark is measured by GPS. We playback the GPS data and process the data at the field by a notebook computer. When the difference of the az- imuths obtained by two measurements of GPS is $<0.1^{\prime}$, we finish the GPS measurement. If the difference of the azimuths obtained by two measurements of GPS is $>0.1^{\prime}$, then we continue the GPS measurements again.

Table 5 shows statistical results of the field observation time $t$ by GPS, the number Ns of the received satellite and the PDOP values in different regions of China in 2003. In Table 5, M is the number of stations, there are altogether 135 stations in China, and the separation between the adjacent stations is $\sim 250 \mathrm{~km}$.

Table 5 gives the value range $a \sim b$, the mean value $m$ and its standard deviation $\sigma(m \pm \sigma)$ for $t$, Ns and PDOP; For example, there are 18 stations in Northeast region of China, the statistical results show that $t$ range is $35 \sim 103$ min, the mean is $52.9 \mathrm{~min}$ and the standard deviation is 21.8 min. The observation results show that when the number of the received satellite is large $(>8)$, the satellite condition is good and the PDOP value is small $(<3.0)$, then the observation time is short ( $20 \mathrm{~min} \sim 30 \mathrm{~min}$ ), otherwise the observation time is long (>30 min). It can be seen from Table 5 that the number of the received satellite is $5 \sim 10$, the average is 7.3 ; the PDOP value is $1.7 \sim 5.4$, the average is 2.7. The field observation time by GPS is $18 \sim 103 \mathrm{~min}$, the average is $45.4 \mathrm{~min}$. Therefore, the GPS field observation should be carried out when the satellite signals are better received.

Table 6 shows the statistical results of the separation $d$ between the station and the reference mark, and the difference $\Delta \alpha$ of the azimuths obtained by 2 measurements of GPS at each station in various regions of China in 2003. In Table $6, \mathrm{M}$ is the number of the stations. Table 6 gives the value range $a \sim b$, the mean value $m$ and its standard deviation $\sigma(m \pm \sigma)$ for $d$ and $\Delta \alpha$; For example, in Northeast region of China, there are 18 stations, the statistical results 
Table 6. Statistical results of separation d between the station and the reference mark, and the difference $\Delta \alpha$ between the azimuths measured by GPS in various regions of China.

\begin{tabular}{cccc}
\hline Region & $\mathrm{M}$ & $\mathrm{d}(\mathrm{m})$ & $\Delta \alpha$ \\
\hline Northeast region & 18 & $222 \sim 416$ & $0.0^{\prime \prime} \sim 5.9^{\prime \prime}$ \\
North-China region & 18 & $150 \sim 387$ & $0.4^{\prime \prime} \sim 4.7^{\prime \prime}$ \\
Southeast region & 20 & $207 \sim 386$ & $0.1^{\prime \prime} \sim 5.0^{\prime \prime}$ \\
Southwest region & 38 & $201 \sim 582$ & $0.1^{\prime \prime} \sim 3.7^{\prime \prime}$ \\
& & $267 \pm 45$ & $1.4^{\prime \prime} \pm 0.7^{\prime \prime}$ \\
Northwest region & 41 & $162 \sim 280$ & $0.0^{\prime \prime} \sim 2.8^{\prime \prime}$ \\
& & $224 \pm 25$ & $0.9^{\prime \prime} \pm 0.8^{\prime \prime}$ \\
& & & \\
China & & $150 \sim 581$ & $0.0^{\prime \prime} \sim 5.9^{\prime \prime}$ \\
& & $248 \pm 35$ & $1.6^{\prime \prime} \pm 0.8^{\prime \prime}$ \\
\hline
\end{tabular}

show that the range of separation $\mathrm{d}$ between the station and the reference mark is $222 \sim 416 \mathrm{~m}$, the mean is $262 \mathrm{~m}$ and the standard deviation is $43 \mathrm{~m} ; \Delta \alpha$ range is $0.0^{\prime \prime} \sim 5.9^{\prime \prime}$, the mean is $2.0^{\prime \prime}$ and the standard deviation is $0.9^{\prime \prime}$. Generally speaking, the statistical results of the GPS observational data at 135 stations in China show that the separation $d$ between the station and the reference mark is $150 \sim 581 \mathrm{~m}$, the average is $248 \mathrm{~m}$ and the standard deviation is $35 \mathrm{~m}$; The difference $\Delta \alpha$ is $0.0^{\prime \prime} \sim 5.9^{\prime \prime}$, and the average is $1.6^{\prime \prime}$ and the standard deviation is $0.8^{\prime \prime}$. The results in Table 6 show that the azimuths are stable and accurate; they are not related to the space change.

\section{Analysis and Processing for the Measurement Data}

Using $D_{1}$ which is an angle between the geomagnetic north direction and the reference mark measured by DI magnetometer and $D_{2}$ which is an azimuth between the geographical north direction and the reference mark measured by GPS, the geomagnetic declination is obtained: $D=D_{1}-D_{2}$.

The data of geomagnetic declination $D$ at the abovementioned 135 stations in China were reduced and processed by using the geomagnetic data at 35 observatories in China. The reduced values of $D$ at various stations were obtained. The standard deviations of the reduced value were $0.02^{\prime} \sim 0.31^{\prime}$, the average was $0.08^{\prime}$. The results show that the data of geomagnetic declination are accurate.

In order to model the geomagnetic field in China, we used the above-mentioned reduced data, the synchronous observation values at 35 observatories in China and the geomagnetic data calculated from the IGRF (International Geomagnetic Reference Field) (IAGA, 2001) at the 38 points (Fig. 2). The Taylor polynomial model (Gu et al., 2005) and the spherical cap harmonic model (Haines, 1985; An, 2003; Gu et al., 2004, 2006; Gao et al., 2005) of geomagnetic declination in China were established. In calculating the geomagnetic field model, the results appear larger misfit of ge-

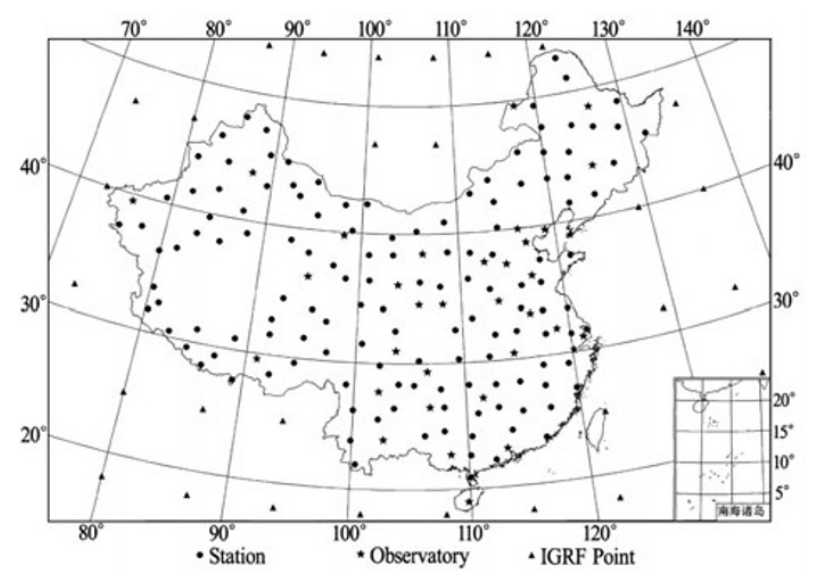

Fig. 2. Distribution of the geomagnetic stations and observatories in China.

omagnetic anomalies in the boundary area when only using the geomagnetic data at 135 stations and 35 observatories in China. After adding the calculation values at some IGRF points in the boundary area, the above-mentioned misfit of geomagnetic anomalies is improved (Gu et al., 2004, 2006; Gao et al., 2005). In order to search for the rationality of the number of the added IGRF points and the distribution, a model calculation was done for some groups of selecting 28 48 IGRF points and the homogeneous distribution. The results for different groups were analyzed and compared. The comprehensive analysis and comparison show that the effects become the best when adding 38 IGRF points as shown in Fig. 2. This shows that it is necessary and rational to add IGRF points appropriately.

Figure 3 gives the 8 -order spherical cap harmonic model of geomagnetic declination in China. It can be seen from Fig. 3 that geomagnetic declinations in the northwest part are positive and those in the northeast part are negative. The mean square deviation between the 8-order spherical cap harmonic model in China and the data of geomagnetic dec- 


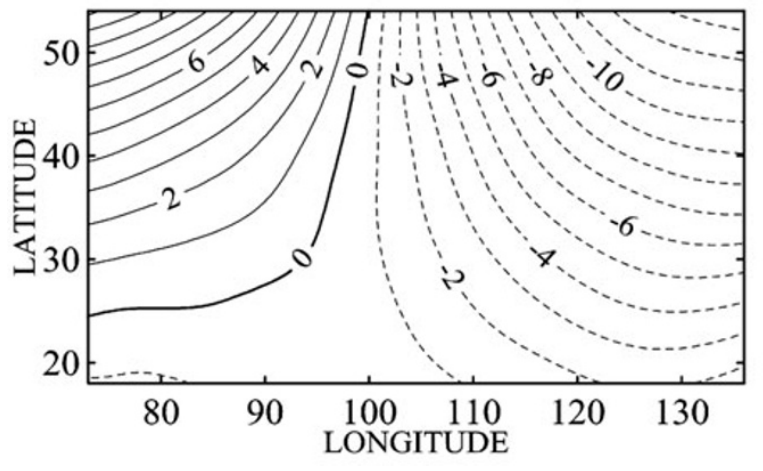

Fig. 3. Spherical cap harmonic model of geomagnetic declination in China (unit: degree).

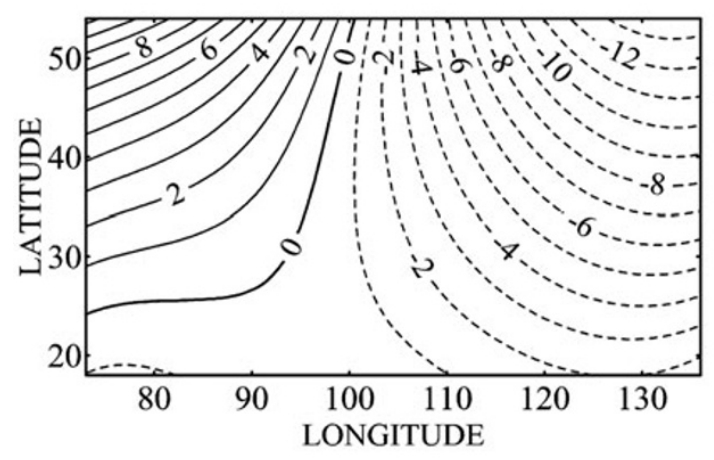

Fig. 4. Taylor polynomial mode of geomagnetic declination in China (unit: degree).

lination is $8.96^{\prime}$. It shows that this model can well represent the space distribution of geomagnetic field in China.

Figure 4 shows the 5-order Taylor polynomial model of geomagnetic declination in China. The mean square deviation between the Taylor polynomial model and the data of geomagnetic declination is $8.65^{\prime}$ which is very closed to the mean square deviation $8.96^{\prime}$ between the spherical cap harmonic model in China and the data of geomagnetic declination. It can be seen from Figs. 3 and 4 that these two models in China are very similar.

\section{Discussion}

In the principle of measuring the azimuth by GPS, the convergence angle $\gamma$ on the plane meridian and the directional correction $\delta$ must be improved in order to change the geodetical azimuth $\beta$ on the ellipsoid surface into the plane coordinate azimuth $\alpha$. In fact, because the separation between the station and the reference mark is only 150 581 $\mathrm{m}$, the improved convergence angle $\gamma$ on the plane meridian is $1^{\prime} \sim 10^{\prime}$, and the improved directional correction $\delta$ is $<0.01^{\prime \prime}$.

Locating errors by GPS come from 4 factors: (a) factor related to the GPS satellites; (b)factor related to the propagation path of signals from the satellites; (c) factor related to the receivers; (d) factor of artificial or calculating influence etc. The practical measurement results show that the Ashtech ProMark2 GPS system is very good for measuring the azimuth in geomagnetic survey.
Table 7 shows the error estimation of the azimuth measured by GPS, where M is the number of GPS observations, $\Delta \alpha$ is the difference between the azimuths measured by different surveys, $\Delta \alpha_{1}$ is the difference between the azimuths measured by GPS and those measured by the astronomical observation (see Table 1), $\Delta \alpha_{2}$ is the difference between the azimuths measured by 10 sets of GPS and the average (see Table 3), $\Delta \alpha_{3}$ is the difference between the azimuths measured by GPS and the average during 2002 2003 (see Table 4), $\Delta \alpha_{4}$ is the difference of the azimuths by 2 measurements of GPS at 135 stations in China in 2003 (see Table 6).

Table 7 gives the value range $\mathrm{a} \sim \mathrm{b}$ for $\Delta \alpha$, the mean value $m$ and the standard deviation $\sigma(m \pm \sigma)$ for $\Delta \alpha_{1}, \Delta \alpha_{2}$, $\Delta \alpha_{3}$ and $\Delta \alpha_{4}$; For example, the $\Delta \alpha_{1}$ range is $0.7^{\prime \prime} \sim 3.8^{\prime \prime}$, the average is $2.5^{\prime \prime}$ and the standard deviation is $1.5^{\prime \prime}$. It is obtained from Table 7 that the mean absolute error of the azimuths measured by GPS is estimated as $2^{\prime \prime} \sim 3^{\prime \prime}$, and the mean relative error is estimated as $1^{\prime \prime} \sim 2^{\prime \prime}$. Therefore, the azimuthal accuracy measured by GPS is estimated as $0.1^{\prime}$. In comparison to the azimuthal accuracy $1.0^{\prime}$ which is given by Newitt et al. (1996), the azimuthal accuracy $0.1^{\prime}$ obtained in this paper is much better.

The GPS measurement results show that in order to get the accurate and reliable data, the distance between the station and the reference mark should be $\geq 200 \mathrm{~m}$; There should be no blocking in the range $>100 \mathrm{~m}$ around the station and the reference mark; There should be a wide field of vision between the station and the reference mark, and small difference of the elevations at the station and the reference mark. We should carry out GPS measurements during the time when the satellite signals are better received.

One of the key problems in establishing the geomagnetic model is to determine the truncation order $K$. The larger the $K$ is, the higher the space resolution is, and the smaller the mean square deviation between the model calculation value and the observation value is. However, when $K$ is large, the model calculation results are relatively unstable. In order to reasonably determine $K, K$ was respectively taken as $1 \sim 12$ for the Taylor polynomial model during the model calculation. The results for various $K(1 \sim 12)$ were analyzed and compared, $K=5$ was finally determined for the Taylor polynomial model. Similarly, for the spherical cap harmonic model, $K$ was respectively taken as $1 \sim 12$ during the model calculation; then the results for various $K(1 \sim 12)$ were analyzed and compared, $K=8$ was finally determined (Gu et al., 2004, 2006).

The Taylor polynomial model of geomagnetic field is convenient in calculation and application. However, the deficiency of Taylor polynomial model is that it does not meet the potential theory of geomagnetic field and can only express the two-dimensional distribution of geomagnetic field. The spherical cap harmonic model of geomagnetic field is complicated in calculation, but it can meet the potential theory of geomagnetic field and express the threedimensional structures of geomagnetic field. Figures 3 and 4 show that these two models in China for 2003 are very similar. Therefore, these two models have good consistency and complementation. Different users can choose one from the two models to meet their own demand. 
Table 7. Error estimation of the azimuths measured by GPS.

\begin{tabular}{ccccc}
\hline & $\Delta \alpha_{1}$ & $\Delta \alpha_{2}$ & $\Delta \alpha_{3}$ & $\Delta \alpha_{4}$ \\
\hline M & 4 & 10 & 5 & 135 \\
$\Delta \alpha$ & $0.7^{\prime \prime} \sim 3.8^{\prime \prime}$ & $0.1^{\prime \prime} \sim 5.1^{\prime \prime}$ & $0.4^{\prime \prime} \sim 3.7^{\prime \prime}$ & $0.0^{\prime \prime} \sim 5.9^{\prime \prime}$ \\
Mean & $2.5^{\prime \prime} \pm 1.5^{\prime \prime}$ & $2.7^{\prime \prime} \pm 1.9^{\prime \prime}$ & $2.9^{\prime \prime} \pm 1.4^{\prime \prime}$ & $1.6^{\prime \prime} \pm 0.8^{\prime \prime}$ \\
\hline
\end{tabular}

\section{Conclusions}

The application of GPS in geomagnetic survey is to measure the azimuth and locate the geomagnetic station and site. The GPS tests and the GPS measurements at 135 stations in China during 2002 2003 show that the time when the satellite signals are better received is just the better condition of measuring the azimuth by GPS. The character parameters of the better condition are larger number of the received satellites $(>6)$ and less PDOP value $(<3.0)$. The azimuthal accuracy measured by GPS is $0.1^{\prime}$ in this paper.

Comparing with the traditional astronomical observation, GPS is automatic observation and can greatly decrease the influence of weather on geomagnetic survey. It can save the survey time and raise the efficiency of field work by using GPS. Therefore, it is very effective and very convenient to use GPS in measuring the azimuth.

Acknowledgments. This research was supported by the National Natural Science Foundation of China (40436016), the Ministry of Sciences and Technology of China and the China Earthquake Administration. We thank Prof. Hisashi Utada and Prof. Toshihiko Iyemori for their helps and good suggestions. We also appreciate the two referees for very useful comments. Contribution No. 05FE3014 of Institute of Geophysics, China Earthquake Administration.

\section{References}

An, Z., Spherical cap harmonic analysis of the geomagnetic field and its secular variation in China for 2000, Chinese J. Geophys., 46(1), 85-91, 2003.

Caporali, A., S. Martike, and M. Massironi, Average strain rate in the Italian crust inferred from a permanent GPS network, Geophys. J. Int., 155(1), 254-268, 2003.

Fernandez, J., F. J. Gonzalez-Matesaz, J. F. Prito, G. Rodriquez-Lelasco, A. Staller, A. Alonso-Medina, and M. Charco, GPS monitoring in the $\mathrm{N}-\mathrm{W}$ part of the Volcanic Island of Tenerife, Canaries, Spain, Pure Appl. Geophys., 161(7), 1359-1377, 2004.

Gao, J., Z. An, Z. Gu, W. Han, Z. Zhan, and T. Yao, Selection of geomagnetic normal field and calculation of geomagnetic anomalous field, Chinese J. Geophys., 48(1), 66-73, 2005.

Gu, Z., Z. An, J. Gao, W. Han, and Z. Zhan, Spherical cap harmonic analysis on the geomagnetic field in Beijing-Tianjin-Hebei region, Chinese J. Geophys., 47(6), 1128-1134, 2004.

Gu, Z., Z. Zhan, J. Gao, W. Han, Z. An, and T. Yao, Geomagnetic survey and geomagnetic model in China for 2003, in Proceedings of the XIth IAGA Workshop on Geomagnetic Observatory Instruments, Data Acquisition and Processing, edited by K. Yumoto and H. Utada, 338 pp., November 9-17, 2004, Kakioka and Tsukuba, Japan, 2005.

Gu, Z., Z. Zhan, J. Gao, W. Han, Z. An, T. Yao, and B. Chen, Geomagnetic survey and geomagnetic model research in China, Earth Planets Space, 58, this issue, 739-748, 2006.

Haines, G. V., Spherical cap harmonic analysis, J. Geophys. Res., 90(B3), 2583-2592, 1985.

Hori, H., T. Kameda, and T. Kato, Prediction of stress field in Japan using GPS network data, Earth Planets Space, 52(11), 1101-1106, 2000.

$\mathrm{Hu}$, J., S. Yu, J. Angelier, and H. Chu, Active deformation of Taiwan from GPS measurements and numerical simulations, J. Geophys. Res., 106(B2), 2265-2280, 2001.

IAGA Division V, Working Group 8, International Geomagnetic Reference Field 2000, Phys. Earth Planet. Inter., 120, 39-42, 2001.

Jankowski, J. and C. Sucksdoff, Guide for Magnetic Measurements and Observatory Practice, International Association of Geomagnetism and Aeronomy, 216 pp., 1996.

Koketsu, K., S. Yoshida, and H. Higashihara, A fault model of the 1995 Kobe earthquake from the GPS data on the Akashi Kaikyo Bridge and other datasets, Earth Planets Space, 50(9), 803-811, 1998.

Kong, X. and S. Mei (ed.), Control Geodesy, Press of Wuhan Geodesy University, Wuhan, China, 386 pp., 1996 (in Chinese).

Ma, Z., X. Chen, and S. Ye, Research on the present crust movements in the Chinese mainland by GPS, Science Bulletin, 46(13), 1118-1120, 2001.

McClusky, S., R. Reilinger, S. Mahmoud, D. Ben Sari, and A. Tealeb, GPS constraints on Africa (Nubia) and Arabia plate motions, Geophys. J. Int., 155(1), 126-138, 2003.

Miura, S., S. Ueki, T. Sato, K. Tachibana, and H. Hamaguchi, Crustal deformation associated with the 1998 seismo-volcanic crisis of Iwate Volcano, Northeastern Japan, as observed by a dense GPS network, Earth Planets Space, 52(11), 1003-1009, 2000.

Newitt, L. B., C. Barton, and J. Bitterly, Guide for Magnetic Repeat Station Surveys, the International Association of Geomagnetism and Aeronomy, 112 pp., 1996.

Nishimura, S., M. Hashimoto, and M. Audo, A rigid block rotation model for the GPS derived velocity field along the Ryukyu arc, Phys. Earth Planet. Inter., 142(3-4), 185-203, 2004.

Nocquel, J. M. and E. Calais, Geodetic measurements of crustal deformation in the Western Mediterranean and Europe, Pure Appl. Geophys., 161(3), 661-681, 2004.

Wang, W., Y. He, and Z. Yao, Complexity of the coseismic rupture for 1999 Chi-Chi earthquake (Tainwan) from inversion of GPS observations, Tectonophysics, 382(3-4), 173-187, 2004.

Yu, J. and C. Zhang, GPS-gravity boundary problem, China Sciences (D), 33(10), 988-996, 2003.

Zhou, J., W. Huang, A. Chen, X. Gao, P. Qiu, L. You, H. Nie, and X. Zhao, Azimuth measurement and error analysis at Beijing geomagnetic observatory, Seismological and Geomagnetic Observation and Research, 18(6), 73-79, 1997 (in Chinese).

Zhu, W., Y. Fu, X. Zhang, Q. Wang, and S. Wang, Horizontal strain field and tectonic deformation revealed by GPS primary results, Chinese J. Geophys., 46(5), 609-615, 2003.

Z. Gu (e-mail: guzwg@sohu.com), Z. Zhan, J. Gao (e-mail: gaojt2000@yahoo.com.cn), T. Yao, and W. Han (e-mail: whan@263.net) 